\title{
SBH AND THE INTEGRATION OF COMPLEMENTARY APPROACHES IN THE MAPPING, SEQUENCING, AND UNDERSTANDING OF COMPLEX GENOMES
}

\author{
Radoje Drmanac, Snezana Drmanac, Ivan Labat, Aleksandra Vicentic, \\ Anne Gemmell, Nick Stavropoulos, and Jonathan Jarvis \\ Integral Genetics Group \\ Biological and Medical Research Division \\ ANL/BIM/CP-75997 \\ Argonne National Laboratory, Argonne, Illinois 60439, U.S.A. DE93 004196
}

\begin{abstract}
A variant of sequencing by hybridization (SBH) is under development with a potential to inexpensively determine up to 100 million base pairs per year. The method comprises five experimental steps: 1) arraying short clones in 864-well plates; 2) growth of the M13 clones or PCR of the inserts; 3 ) automated spotting of DNAs by corresponding pin-arrays; 4) hybridization of dotted samples with $200-3000{ }^{32} \mathrm{P}$ - or ${ }^{33} \mathrm{P}$-labeled 6- to 8mer probes; and 5) scoring hybridization signals using storage phosphor plates. The method opens up intriguing possibilities for genome analysis. Some 200 7- to 8-mers can provide an inventory of the genes if cDNA clones are hybridized, or can define the order of $2-\mathrm{kb}$ genomic clones, creating physical and structural maps with 100-bp resolution; the distribution of G+C, LINEs, SINEs, and gene families would be revealed. cDNAs that represent new genes and genomic clones in regions of interest selected by SBH can be sequenced by a gel method. Uniformly distributed clones from the previous step (20\% of all) will be hybridized with 2000-3000 6- to 8-mers. As a result, approximately $50-60 \%$ of the genomic regions containing members of large repetitive and gene families and those families represented in GenBank would be completely sequenced. In the less redundant regions, every base pair is expected to be read with 3-4 probes, but the complete sequence can not be reconstructed. Such partial sequences allow the inference of similarity and the recognition of coding, regulatory, and repetitive sequences, as well as study of the evolutionary processes all the way up to the species delineation. Targeted gel sequencing with up to $10 \%$ error can be effectively used to complete sequences of genomic segments more than $70 \%$ similar to the treated ones. A 1000-bp read from a single gel strip would be sufficient to complete several thousand base pairs of sequence generated by duplications or present in genomés of closely related species. More interestingly, partial sequences generated with the same probes on three to four 70 - to $90 \%$-similar genomes may mutually complete each
\end{abstract}
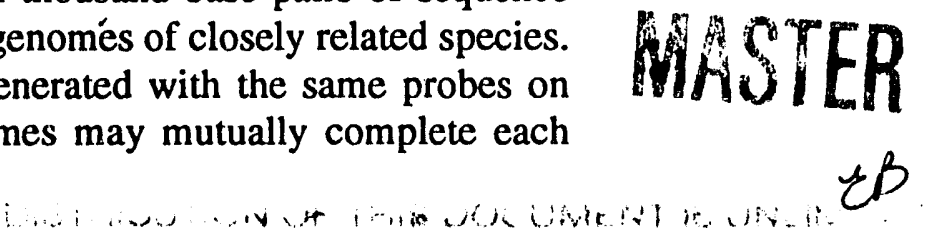
other, minimizing additional experimental data. This SBH variant can effectively fill a gap between expensive 300 - to 600 -bp runs on sequencing gels and the $10-$ to $100-\mathrm{kb}$ resolution of the presently available mapping techniques.

\section{Introduction}

Nucleic acid hybridization process ${ }^{1}$ is frequently used in cloning, mapping, and sequencing procedures. ${ }^{2-7}$ Sequencing by hybridization (SBH) is an ultimate application of the hybridization reaction. A sufficient number of chemically synthesized oligonucleotides of known sequence hybridized with target DNA under conditions that discriminate full matches from mismatches can reveal the target DNA sequence. Five teams are claiming independent invention of SBH in 1987 and 1988..$^{8-13}$ Two experimental formats are possible. In format 1 , individual probes are hybridized to genomic DNA (or cDNAs) attached to a support. ${ }^{13}$ In format 2 , genomic fragments (or cDNAs) are labeled and separately hybridized to a array of oligonucleotides. ${ }^{10}$ Format 1 is more efficient for complex genome sequencing, in which the number of clones is much larger than the number of probes. Format 2, at the present level of development, is more appropriate for sequencing a small number of short DNA fragments. Substantial array miniaturizations in both formats ("sequencing chips") are envisioned. ${ }^{14-18}$

In this paper we will discuss the usefulness of short oligonucleotide hybridization assays for ultrafine physical and structural mapping and for partial to complete DNA sequencing. The first SBH format 1 data production line will be reviewed, emphasizing applications for cataloging genes by producing an oligonucleotide sequence signature $(\mathrm{OSS})^{19}$ of random cDNA clones, 100-bp resolution mapping by ordering short clones, recognition of the genes, and accelerated simultaneous sequencing of similar genomes. An intriguing possibility of species recognition on the basis of partial sequences and some requirements for an efficient analysis of complex genomes will also be discussed.

\section{The First SBH Data Production Line}

We are building a production line based on many standard procedures of molecular biology and recombinant DNA technology, with a capacity to score several million hybridization assays of 6- to 8-mer probes per day. Detailed descriptions of the steps of clone preparation by growing M13 or by PCR, high-density spotting of DNA samples by robotic printing using metal pin arrays, and discriminative hybridization of short oligonucleotide probes have been provided. ${ }^{20}$

Clones (1-2 kb) are arrayed by picking or by limit dilution in 864-well plates (2-mm well diameter; Helix Inc., Calif.). Preparative plates are inoculated using a corresponding pin array, and, after growth (in the case of M13) or PCR amplification (in the case of other vectors) using BioOvens (BioTherm Corporation, Fairfax, Virginia), the 
DNA is spotted on nylon membranes. We adapted a Biomek1000 tablet, pin tool, and software to be able to transfer a sufficient amount of DNA by repeated spotting in the same dots, to achieve a high dot density by offsetting up to 324 plates, and to generate production size filters which accommodate an array of $2,3,4$, or 6 plates. Filters are hybridized in boxes with semiautomatic washing procedure using ${ }^{32} \mathrm{P}$ - or ${ }^{33} \mathrm{P}$-labeled probes. On the basis of the content and distribution of $\mathrm{G}+\mathrm{C}$, probes are divided into five groups, and for each group the appropriate probe corsentration and washing time and temperature are defined. ${ }^{20}$

Hybridization images are obtained using storage phosphor plates. The plates are scanned on a PhosphorImager (Molecular Dynamics, Sunnyvale, Calif.). The technology has a space resolution of $300 \mu \mathrm{m}$ for ${ }^{32} \mathrm{P}^{20}$ and probably $100 \mu \mathrm{m}$ for ${ }^{33} \mathrm{P}$ ( $\mathrm{R}$. Crkvenjakov, personal communication and ref. 21). By spotting 324 plates (96-well) or thirty-six 864well plates using $0.25-\mathrm{mm}$ pins, the distance between dots will be about $250 \mu \mathrm{m}$, which is above the space resolution of ${ }^{33} \mathrm{P}$. At this density, 186,624 dots can be accommodated on a 6-pattern filter. Images of over 100 such filters can be generated per eight working hours, allowing a throughput exceeding 15 million clone-probe scores per day. Our working density is eighty-one 96-well plates, and the present hybridization set-up has a throughput of $20 \%$ of the PhosphorImager capacity, allowing the scoring of one million clone-probe signals per day.

\section{Image Analysis and Data Storage}

Recently a software program (DOTS) for the analysis of hybridization images of the densely arrayed dots was developed by one of us (J.J.). The DOTS program extracts the magnitude and location of probe-to-clone hits with nearly minimal operator assistance. The input data to DOTS consists of a large 3- to 20-megabyte binary image file created by a Molecular Dynamics PhosphorImager on a DOS platform. The large size of the input files is a result of writing two bytes per pixel as unsigned short integers from 0 to 65,535 . The PhosphorImager scan density is 114 rows per centimeter, which results in about 17 pixels between dot centers spotted in a $6 \times 6$ pattern using 36 microtiter plates, each plate containing 96 wells with $9-\mathrm{mm}$ spacings. The DOTS program output condenses 578 bytes per dot to 8 bytes per dot, as peak value, mean value, peak offset, and center of mass offset.

The first step in the DOTS program is to find approximate filter edges. This is done by summing short lengths of pixels near each corner. A periodic pattern of peaks and valleys is obtained which indicates the location of each outermost row or column near the corners, and then by extrapolation the respective corners. After the four preliminary corners are assigned, a parallelogram is formed by pushing out the corner having the most obtuse inner angle, to obtain a correct fit even if one corner is defined incorrectly. Then a skewed rectangle is formed by shrinking the longer diagonal. In rare cases where on 
two or more corners there is no sufficient number of positive dots, the operator may intervene manually to adjust the fit, with special emphasis on the skew angle.

Next, a scan of the image provides sums of pixels of rows and columns in the skewed angle directions. Data is loaded and shifted in RAM memory to minimize physical disk access. The pixel sums produce a recognizable pattern of peaks and valleys (Fig. 1). Depending upon the quality of the image, the DOTS program will intelligently interpolate to find ambiguous peaks. An optimized set of row and column vectors is obtained which maps the target centar of each dot without reliance upon local dot topography, the main problem with early DOTS program versions.

Finally, dot values are extracted by drawing an imaginary square around each dot, centered at the optimized row/column target. The imaginary square edge length is typically $3 / 4$ of the dot span. The true peak pixel value within the target area is reported as the dot value unless the peak value occurs on the target edge, in which case the average dot value is reported. This corrects for spreading radiation effects due to a strong neighboring dot.

The density of eighty-one 96-well plates per a filter can be successfully analyzed with the present version of the DOTS program. Future plans are to implement batch capability to analyze multiple filters from the same image; to process filters with 324 plates; to port the Borland C code to UNIX; and to convert the output to the format of Sybase, a relational data base shared by most human genome centers.

To ensure automatic data entry in the data base, three levels of coding and decoding are envisioned. Storing and preparative plates are to be labeled by bar code, which has to be read on a robotic station at the moment of preparative plate inoculation or sample spotting. Filters are to be labeled by a dot code using various complex DNAs that can be positively scored by any 6- to 8-mer probe. Finally, the identity of the hybridized probe will be decoded on the basis of sequenced DNA samples. The pattern of positive and negative signals with hundreds of the control samples spotted on each membrane will reveal sequence of the probe.

For proper storing of clone-probe results in the data base, about 10 bytes is necessary. Assuming a steady throughput of 5 million clone-probe scores per day and 200 working days in a year, 10 gigabytes of row data can be accuinulated in a year. Obviously, a high-capacity system for data storing and efficient relirieving has to be developed. Depending which clone and probe sets are used, different genetic information can be extracted from the data. Some of the applications will be discussed in the following sections. Due to large sets of possible pairwise comparisons, a sophisticated software package is required for gene identification, defining high resolution maps and sequence assembly. 


\section{Cataloging Genes}

The most exciting application of the developed data production line is the analysis of expressed sequences. The projects for partial sequencing of random cDNA clones by gel methods are producing first results. ${ }^{22.23}$ The importance of partial sequencing of cDNA and possibilities of applying oligonucleotide hybridization for that purpose have been described. ${ }^{24,19}$ The advantages of SBH are the capability of efficient analysis of millions of the clones and determination of sequences which are distributed more uniformly over the entire length of the clones. Disadvantages are the lack of continuous sequences necessary to design PCR primers and to decode amino acid sequences, when a small number of probes is used.

We are accumulating hybridization data for 100 probes on up to 100,000 cDNA clones from a three-month-postnatal human brain cDNA library (b4HB3MA) kindly provided by Dr. Marcelo Bento Soares (Columbia University). DNA samples have been prepared by PCR in BioOvens. The probe set consists of several very distinct subsets; for example noncoding, Alu-specific, coding, motif-comprising, and similar subsets will be used. Different probe sizes ( 6 to 8 bases, and in some cases, 9 and 10 bases) are necessary to ensure an appropriate frequency for clones varying in size and nucleotide composition. A few sets of overlapping oligonucleotides are necessary for discerning among overlapped and similar clones. Hybridization results of a 6-mer and a 7-mer with cDNA clones are presented in Fig 2. The frequency of positive dots is much higher with the GC reach 6-mer due to statistically expected four times.higher frequency in random sequences and due to average GC of $60 \%$ in coding sequences. The frequency is high, even the probe nngcggcgnn contains two CpG dinucleotides, known to be deficient in eukaryotic DNAs.

A software package is under development for extracting genetic data from the relative intensities of hybridization signals among the clones. The intensities have to be normalized for variation in amount and complexity of DNA among the clones and for variation in stability and hybridization condition among the probes. The resulting values create the signatures of clones, and allow estimation of the similarity among the pairs of the analyzed clones as well as among the clones and the sequences of known genes. We expect to define 20,000 new genes and over 100 new, distinctive gene families. Cataloging disting genes and having access to the related clones represent an ultimate normalization of the cDNA libraries. If successful, the experiment will be repeated on over a million clones combined from a few tissues in order to make an inventory for most of the genes and to get an overall insight into the gene expression pattern.

\section{Physical Mapping of Complex Genomes with 100-bp Resolution}

Physical mapping with a resolution higher than $10,000 \mathrm{bp}$ is not considered for complex genomes. Even restriction mapping of a particular gene locus is performed with 
a precision of a few $\mathrm{kb}$. Our hybridization data production line offers a possibility for efficient production of ultrafine maps, having an average resolution of $100 \mathrm{bp}$ (Fig. 3), by applying the link-up strategy developed by $\mathrm{H}$. Lehrach for cosmid mapping. ${ }^{5.25}$ One to two kb inserts (cloned in M13 or prepared by PCR) spotted on membranes and hybridized with 6- to 8-mer probes, will provide a high frequency of positively scored clones (30-50\%). Successful hybridization of short probes and simple preparation of pure DNA samples eliminates major problems related to the fingerprint of cosmid libraries. In the latter case, bacterial DNA allows only specific probes to be used. ${ }^{26}$ In collaboration with B. Koop and L. Hood of Caltech, we are trying to determine the map for a human cosmid by fingerprinting $1400 \mathrm{M} 13$ subclones of the cosmid DNA with 200 7- to 8-mer probes. A heuristic algorithm for clone ordering is under development. The concept of the algorithm will be described in the accompanying paper. The basic usefulness of such a map is to provide clones and information which may significantly reduce redundancy and thus simplify genome sequencing. In the next section, an intriguing utility of ultrafine physical maps will be described.

\section{Structural Genome Maps and Partial Sequences}

Ordinary physical maps consist of knowledge of the order and distances among the markers (restriction sites and fragment, clones, STSs). There is little information about compositional characteristics and structural elements in the DNA between markers. However, when density of the markers is high and they reveal segments of the sequence (for example, restriction enzyme cutting sites), compositional characteristics of the genomic DNA become apparent. The genomic maps having these characteristics have been called structural maps. ${ }^{27,28}$ Structural maps contain information about the order and positions of the main genome structural elements such as repetitive sequences or genes.

Mismatch discriminative hybridization of 6- to 8-mers with overlapped genomic clones 1 - to $2-\mathrm{kb}$ long offers a very efficient way for creating structural maps. Densely overlapped (10 genome equivalents) 1 - to 2 -kb clones define consecutive segments called informational fragments (IFs) ${ }^{13}$ that are 100 bp long, on average (Fig. 3). If appropriate probes are used, the amount of sequence information can be sufficient for recognition of major structural elements of genomic DNA.

A structural map is obtained by a simulation experiment on $350 \mathrm{~kb}$ of known human sequences using 2007 -mer and 8-mer probes divided into 3 subsets (Fig. 4). The probes reveal $\mathrm{C}+\mathrm{G}$ content (black and white areas generated with probes containing only A or $\mathrm{T})$. The most interesting pattern is obtained with the third group of probes. These probes are selected as the most frequent 7 -mers having more than $2 \mathrm{C}+\mathrm{G}$ bases. As expected, the probes are specific for Alu-repetitive sequences (columns of points). Preliminary results show that these probes also reveal some "Alu-like" senuences or scrambled Alu's. At least four such sequences are found in the beta-globin locus. The 
pattern of Alu repeats can be used as a indicator of the gene positions. As the picture demonstrates, the regions with the minimal similarity to Alu repeats contain genes.

The number of probes from these three groups can be reduced and additional groups can be used. Exon-specific probes represent a very important group. In collaboration with E. Uberbacher (Oak Ridge National Laboratory), we are defining a set of these probes. In a preliminary analysis, we have found that the probes containing $(A, T) C G(A, T)$ sequence are significantly more frequent in coding than in noncoding segments.

Fragments $1-2 \mathrm{~kb}$ in size are easy to clone, more stable than longer fragments, and simple to produce in quantity by plasmid or M13 growth or by PCR, which is becoming the method of choice. Also, the DNA can be spotted in more dense arrays relative to colony or plaque arrays. Finally, oligonucleotide probes do not require any purification and are very easy to label. By combining all of these advantages, obtaining a structural map of human genome can be more straightforward than defining lowresolution maps. Twenty-five million $2-\mathrm{kr}$ clones with $70 \%$ success in preparation will provide 12 genome equivalents, sufficient tor complete coverage of all clonable genome segments. The described production line (ref. 20 and this paper) allows collecting informaton from 300 probes on this number of clones in 3-4 years in a laboratory. By dividing the job among 3-4 laboratories on a chromosomal basis, the structural map of the human genome can be produced in a year, generating a resource of clones and information for further sequencing and advanced studies in molecular genetics.

The next level in revealing genetic information is provided by partial sequencing. ${ }^{24}$ In structural mapping by 3007 -mers, $15 \%$ of the sequence will be read (one positive 7 mer on every $50 \mathrm{bp}$ ). By increasing the number of the probes to the point that most (or all) of the base pairs are scored by a probe (about 20007 -mers), the new possibilities are apparent even if the positively hybridized probes can not be uniquely ordered in a continuous sequence. This density of information allows a precise inference of similariiy among the genome segments, and detection of polymorphic mutated sites and functional motifs. ${ }^{24}$ Very few features can not be decoded on this level (such as open reading frames). Various experimental methods can be undertaken to obtain a complete sequence; for example, additional hybridizations or restriction mapping. An intriguing cost- and speed-effective possibility is the integration of hybridization and gel sequence data from overlapped and similar sequences in and between the genomes. ${ }^{27}$

\section{A Phylogenetic Perspective of Genome Sequencing}

There is a consensus among molecular biologists on the importance of comparing sequences between species. In addition to the possibility of comparative studying, similar genomes can be used to increase sequencing efficiency. The sequencing effort per a genome can be significantly smaller if a few closely related genomes are analyzed 
simultaneously. We recognize three ways to reduce the effort. First, knowledge of the sequences of one genome allows the defining of primers which can work for the other closely related genomes. ${ }^{29}$ Second, the number of the clones in shotgun sequencing approaches can be reduced. Third, and specific for $\mathrm{SBH}$, overlapped clones ${ }^{13}$ and differences between homologous sequences aid in the resolution of branching points. ${ }^{27.28}$ Determining clone order and the assembly of sequence contigs depend on a significant overlap of clones or sequence reads, respectively. Clones and sequences from similar genomes can be used as mutual linking elements. This can significantly extend the contigs, reducing need for complementary directional methods. Our estimate is that two genomes can be sequenced for approximately the effort of one.

The mutual benefits are much larger for the SBH method. The basic problem in sequencing fragments longer than 100 bp by $\mathrm{SBH}$ is the requirement for an enormous redundancy of hybridization data. To sequence a 1000-bp fragment, about 4 million ordinary 11-mer probes are required, e.g. 4000 scores per base pair. In gel sequencing, four scores (one positive and three negative) are in principle sufficient. By a proposed solution which relies on gapped probes, tenfold less redundant data can suffice. ${ }^{10,30} \mathrm{We}$ have measured the efficiency of similar sequence approaches (see Labat and Drmanac, in this book). There is an exponential growth of resolving power by increasing the number of the mutually similar sequences. Integrated data from four related genomes may be sufficient for sequencing 1000-bp fragments by 2000-3000 6-mers or 7-mers. The redundancy is reduced to 5-10 scores per base pair, assuming 50-70\% overlap between clones. This means that the described potential throughput of 15 million cloneprobe assays per day is equal to 1 million bp/day, or 100 million ty/year.

The optimal similarity range $70-90 \%$ is broadly present among genomic sequences (E. coli and Salmonella genomes are $85 \%$ similar; the human and rhesus monkey genomes, 90\%). In addition to the integration of SBH format 1 data from overlapped and similar sequences, targeted complete sequencing of the recognized regions of interest can be performed using gel sequencing or hybridization with a complete oligonucleotide array (SBH format 2). The knowledge of a continuous sequence, even with $10 \%$ error, resolves the branching points in the sequences similar to it. In the recent simulation experiments with 6-mer data, we have successfully reconstructed 300-bp segments more than $70 \%$ similar to known sequences even though the segments are connected to $200-400 \mathrm{bp}$ of unrelated sequences. Thus, by combining the targeted and global sequencing methods on related genomes, a simultaneous sequence determination and decoding process can be performed with an enormous efficiency.

\section{Sequence Convertibility Index and Species Identification}

Sequences of the nucleic acid molecules have been increasingly used for solving phylogenetic questions, e.g., relationships and distances between species or populations. ${ }^{34,35}$ The main problem is the limited amount of sequence information for 
comparative studies. Usually only sequences of one gene or part of one gene are compared. Also, some of the studies are based on molecules that are not common for all living world (rRNAs cannot be used for viral systematics). An efficient way to overcome the lack of data is to determine partial sequences over large genomic regions in many species by applying fast and inexpensive "genome scanning" by oligonucleotide hybridization. In addition, such data can be used for delineation between species and populations or clones of organisms.

We have developed a species concept universal for entire living world (R. Crkvenjakov and R. Drmanac, manuscript submitted). Assuming that genome programs (G) of parents represent the initial conditions (C) for offspring genome programs, a chain of the organisms can be represented as

C0G1 $\rightarrow \mathrm{C} 1 \mathrm{G} 2 \rightarrow \mathrm{C} 2 \mathrm{G} 2$ (no genome change occurs) $\rightarrow \mathrm{C} 2 \mathrm{G} 3 \rightarrow \rightarrow \rightarrow$.

Some changes in genome sequence will not be compatible with parental initial conditions. For example, changes of the $\mathrm{G} 2$ leading to $\mathrm{G} 4$ may not be compatible with $\mathrm{C} 2$. In some cases, reversible changes on parent type may not be compatible with related initial conditions (for example in hypothetical $\mathrm{C} 2 \mathrm{G} 1$ organisms).

The species as natural category can be defined on the basis of the irreversibility principle. A species consists of organisms having genomic sequences convertible each to other through the reproduction cycles. Convertibility means a possibility that two sequences become identical, but not necessarily by physical interchange. Reproductive isolation of sexual organisms can be seen as a phenotypic expression of this principle. As a consequence, some sequence differences between organisms belonging to different species can not be dismissed (converted) through reproduction process. Defining such differences is equal as fundamental understanding of the species category, and it is a very difficult task requiring knowledge of the genome sequences and their function. One the other hand, an estimate of whether such sequence differences exist can be performed with limited knowledge of both genome sequences and sequence functions.

A measure which we call the Sequence Convertibillity Index (SCI) can be defined for the best estimate of the existence of irreversible differences between genomic sequences and consequently, recognition of whether a group of organisms is a distinct species. First of all, differences over the whole genome have to be taken into account. Second, not only average similarity, but also distribution and the types of differences are weighting factors. In this concept, horizontally transferred fragments of genomic DNAs among the organisms are only a additional indicator of whether there is or not a species boundary. SCI can be a very useful parameter, especially for systematics of asexual species. It is likely that partial sequencing by oligonucleotide hybridization can allow its routine measurement. Using genome sequences as a basic source of information and 
having a method enabling researchers to sequence many genomes (even partially) may revolutionize theory and practice of evolutionary biology. .

\section{Integral Genetics: an Efficient Approach to Studying Complex Genomes}

It seems that the most efficient approach to study complex genomes must be based on obtaining, and sophisticated analysis of a sufficient amount of sequence information. ${ }^{16,28}$ This does not mean that only complete and errorless sequences have to be used or that various experimental strategies for testing the functions of the modified DNAs or proteins will not be necessary. On the contrary, the existing and future knowledge about particular genes and other genome units will simplify the decoding anonymous sequences. A multifactorial analysis of the sequences from related genomes can only define a testable hypothesis with a high probability of correctness. This approach (called integral genetics), ${ }^{16,28}$ is already in use, ${ }^{31,32}$ and an explosion may be expected when billions of base pairs (10-100 times more than what is known now) become available and routinely obtainable.

Due to the physical and informatical complexity of genomes, integral genetics requires the application of complementary approaches with synergistic effects. The main examples are the complementarity of the experimental and computational (theoretical) analysis and the global (low-resolution) and local (high-resolution) mapping and sequencing. Theoretical analysis, modeling and simulations may play a significant (if not dominant) role in genome decoding. Also, it is very important to balance effort on obtaining complete sequences of the restricted regions and a significant density of partial sequence information covering many whole genomes. As discussed above, SBH may efficiently provide the appropriate data.

The results of extensive comparisons of the sequences anticipated in integral genetics can be conditionally divided on similarities and differences. Statistically significant similarities (patterns, consensus sequences) efficiently reveal probable functions of the sequence units. ${ }^{31,33}$ Even when some functional sequences have no statistical significance in a genome, conservation among the genomes exists for most of them due to a low probability of occurrence of new sequences with the same function. How to evaluate the functionality of a sequence difference among the species is a more difficult problem. A statistically significant trend of changes in several related species and among the individuals of a species can be helpful. This mandates individual sequencing or a high- resolution genetic mapping based on extensive sequence data. Also information from the examination of the expression pattern of the genes (for example, by proposed screening random cDNA libraries with 100 probes) may be, useful.

It is legitimate to try to give a probable answer to the question of what are the functional differences between genomic sequences of two phylogeneticaly close species 
(for example humans and chimpanzees) before the sequences of their genomes are determined. By modeling changes of a complex and integrated system, and using knowledge of the genetic (physicochemical) possibilities for generating and changing a complex set of instructions recorded in DNA or RNA sequences (knowledge of the "living-system encoding capacity" of nucleic acids) it may be found that some type of modifications have a high probability to code for species specific phenotype. (These probabilities have to be included as important weighing factors in an expert system for SCI measurement.) Differences among the regulatory elements or those expressed in protein sequences, and differences in the number of the members in the gene or regulatory families represent a few distinct possibilities. Basically, the differences may reflect changes in the interaction among existing elements (requiring modification of some elements) or creating new functions (interactions) by adding some elements (usually by duplication of the existing elements with slight modifications). The last possibility looks more probable for complex and integrated programs changed undirectionally through the reproduction chain. Physicochemical characteristics of nucleic acids allow occurrence of the necessary sequence duplications. The miracles of the living world can be explained more naturally by conceptualization and understanding of the "living-system encoding capacity" of nucleic acids than by the existing theories based on the random changes of an inert recording matter (almost system independent and replaceable with the other polymers composed of four or more different monomers and capable of replication) followed by the natural selection. The analyses of this type are going to play a significant role in integral genetics.

A Concept of Presentation for the Next Bioinformatics, Supercomputing, and Complex Genome Analysis Meeting

1. First useful biological data have been produced by $\mathrm{SBH}$.

A. A few cosmids and one bacterial genome are mapped at 100-bp resolution. B. Tens of thousands of genes and about 100 of new gene families are recognized by hybridizing 100,000 random cDNA clones with 100 6- to 8-mer probes.

2. Improved SBH production line has a capacity equivalent to one million base pairs per day.

3. Comparative analysis of one million cDNA clones from a few tissues and comparative sequencing of four similar bacterial genomes are in progress.

4. The expected changes in the genome project in the light of SBH capacity and type of the data which are going to be generated (parallel analysis of many genomes, probabilistic estimates of the roles, expert systems and multifactorial analysis, new requirements for data bases). 


\section{Acknowledgments}

Work supported by the U.S. Department of Energy, Office of Health and Environment Research, under contract No. W-31-109-ENG-38.

\section{References}

1. C. Schildkraut, J. Marmur and P. Doty, J. Molec. Biol. 3 (1961) 593-617.

2. M. Grunstein and D. S. Hogness, Proc. Natl. Acad. Sci. USA 72 (1975) 39613965.

3. E. M. Southern, J. Molec. Biol. 96 (1975) 503-517.

4. S. V. Suggs, R. B. Wallace, T. Hirose, E. H. Kawashima and K. Itakura, Proc. Natl. Acad. Sci. USA 78 (1981) 6613-6617.

5. A. Poustka, T. Pohl, D. P. Barlow, G. Zehetner, A. Craig, F. Michiels, E. Ehrich, A.-M. Frrischauf and H. Lehrach, Col Spring Harbor Symp. Quant. Biol. 51 (1986) 131-139.

6. G. A. Evans and K. Lewis, Proc. Natl. Acad. Sci. USA 86 (1989) 5030-5034.

7. G. M. Church and S. Kiefer-Higgins, Science 240 (1988) 185-188.

8. R. Drmanac and R. Crkvenjakov, Yugoslav Patent Application 570 (1987).

9. E. Southern, United Kingdom Patent Application GB 8810400 (1988).

10. W. Bains and G. C. Smith, J. Theor. Biol. 135 (1988) 303-307.

11. S. C. Macevicz, International Patent Application PC US89 04741 (1989).

12. Y. P. Lysov, V. L. Florentiev, A. A. Khorlyn, K. R. Krhapko, V. V. Shick and A. D. Mirzabekov, Dokl. Adad.Nauk SSSR 303 (1988) 1508-1511.

13. R. Drmanac, I. Labat, I. Brukner and R. Crkvenjakov, Genomics 4 (1989) 114128.

14. R. Drmanac and R. Crkvenjakov, Yugoslav patent application (1989) 767/89.

15. R. Drmanac, Z. Strezoska, I. Labat, S. Drmanac and

R. Crkvenjakov, DNA and Cell Biology 9 (1990) 527-534.

16. R. Drmanac, I. Labat, Z. Strezoska, T. Paunesku, D. Radosavljevic, S. Drmanac and R. Crkvenjakov, eds. C. R. Cantor and H. A. Lim, Electrophoreses, Supercomputing and the Human Genome (World Scientific, Singapore, 1991), pp. 47-59.

17. S. P. A. Fodor, J. L. Read, M. C. Pirrung, L. Stryer, A. T.-Lu and D. Solas, Science 251 (1991) 767-773.

18. K. R. Khrapko, Yu. P. Lysov, A. A. Khorlin, I. B. Ivanov, G. M. Yershov, S. K. Vasilenko, V. L. Florentiev and A. D. Mirzabekov, DNA Sequence-J. DNA Sequencing and Mapping 1 (1991) 375-388.

19. G. G. Lennon and H. Lehrach, Trends Genet. 7 (1991) 314-317. 
20. R. Drmanac, S. Drmanac, I. Labat, R. Crkvenjakov, A. Vicentic and A. Gemmell, Electrophoresis 13 (1992) 566-573.

21. R. J. Zagursky, P. S. Conway and M. A. Kashdan, BioTechniques 11 (1991) 3638.

22. M. D. Adams, J. M. Kelley, J. D. Gocayne, M. Dubnick, M. H. Polymeropoulos, H. Xiao, C. R. Merril, A. Wu., O. Olde, R. F. Moreno, A. R. Kerlavage, W. R. McCombie and J. C. Venter, Science 252 (1991) 1651-1656.

23. A. S. Wilcox, A. S. Khan, J. A. Hopkins and J. M Sikela, Nucl. Acids Res. 13 (1991) 1837-1843.

24. R. Drmanac, G. Lennon, S. Drmanac, I. Labat, R. Crkvenjakov und H. Lehrach, Electrophoreses, Supercomputing and the Human Genome, eds. C. R. Cantor and H. A. Lim (World Scientific, Singapore, 1991), pp 60-74.

25. - H. Lehrach, R. Drmanac, J. Hoheisel, Z. Larin, G. Lennon, D. Nizetic, T. Monaco, G. Zehetner, and A. Poustka, Genome Analysis 1, Genetic and Physical Mapping, Cold Spring Ha:bor Laboratory Press, 1991, 39-81.

26. R. Drmanac, D. Nizetic, A. Beitverda, G. Lennon and H. Lehrach, Nucl. Acids Res. 19 (1991) 5839-5842.

27. R. Drmanac, Genome Mapping and Sequencing, Cold Spring Harbor Laboratory Press, 1992, 318.

28. R. Drmanac and R. Crkvenjakov, Int. J. Genome Res. 1 (1992) 59-79.

29. R. Mazzaella, V. Montanar, J. Kere, R. Reinbold, A. Ciccodicola, M. D'Urso and D. Schlessinger, Proc. Natl. Acad. Sci. USA 89 (1992) 3681-3685.

30. P. A. Pevzner, Yu. P. Lysov, K. R. Khrapko, A. V. Belyavsky, V. L. Florentiev and A. D. Mirzabekov, J. Biomolec. Structure \& Dynamics 9 (1991) 399-410.

31. M. D. Adams, M. Dubnick, A. R. Kerlavage, R. Moreno, J. M. Kelley, T. R. Utterback, J. W. Nagle, C. Fields and J. C. Venter, Nature 355 (1992) 632-634.

32. S. Oliver et al., Nature 357 (1992) 38-46.

33. S. Karlin and V. Brendel, Science 257 (1992) 39-49.

34. M. Novacek, Nature 356 (1992) 121-125.

35. A. Knoll, Science 256 (1992) 622-627.

\section{Figure Legends}

1. Defining positions of the rows and columns of dots. In this figure each peak represents one column of the dots. A slight variation of the distances between the columns is recognizable.

2. Hybridization patterns of cDNA clones. One 7-mer (top) and one 6-mer (bottom) are represented. Accurately fitted grids are drawn automatically and demonstrate the success of the image analysis software. Samples from eighteen 96-well plates are spotted twice, producing pairs of the dots in the strips of six rows. Similar 
signal intensity of the pairs demonstrates the reproducibility of the spotting and hybridization procedures.

3. Parameters of an ultrahigh density map. Long bar represents a chromosome; short bars, clones; and dots (or very short lines on the enlargement), positively hybridized probes.

4. A structural map of human genomic sequences. A model of the human genome comprising $350,000 \mathrm{bp}$ is generated by combining the eight largest known sequences (GenBank entries: Humhprtb, Humghcsa, Humfixg, Humhbb, Humadag, Humalbgc, Humatp1a2, Humbmyh7). Positive finds in 500-bp segments of the sixty 8-mers containing only $A$ or $T$, sixty-six 7-mers containing 1 or $2 \mathrm{C}+\mathrm{G}$, and seventy-four 7-mers containing more than $2 \mathrm{C}+\mathrm{G}$ and having the highest frequency in the combined $350 \mathrm{~kb}$ are represented by white dots. Each $\mathrm{rc}$ : represents a probe. The resolution is five times coarser then in the planned map (see Fig. 3). Positions of the genes in human growth hormone (HGH) and betaglobin (HUMHBB) loci are indicated by vertical bars and position of Line 1 by L. Four new sequences (x) similar to Alu repeats recognized up to now (.) are revealed in the globin locus. 


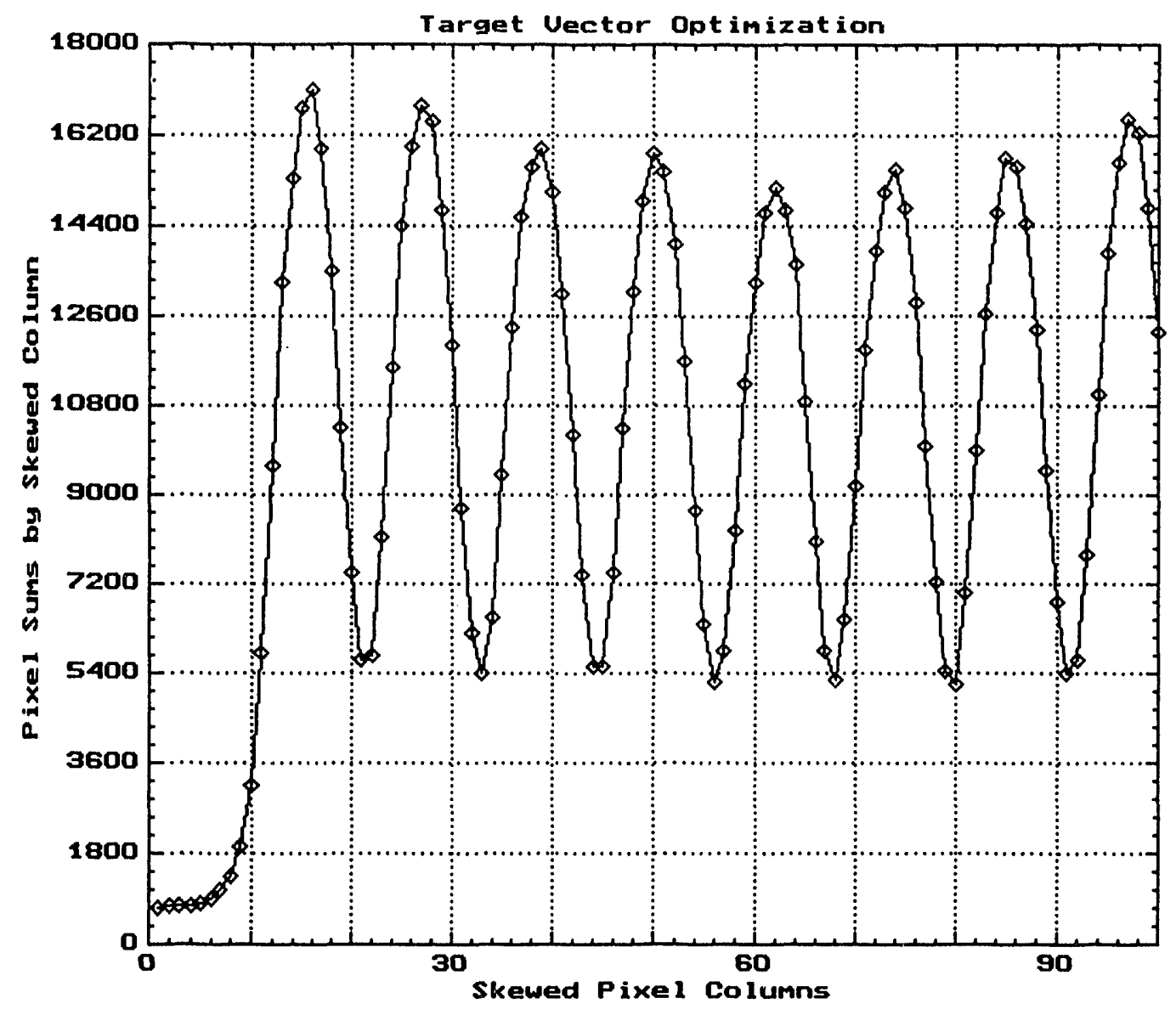

Fig 

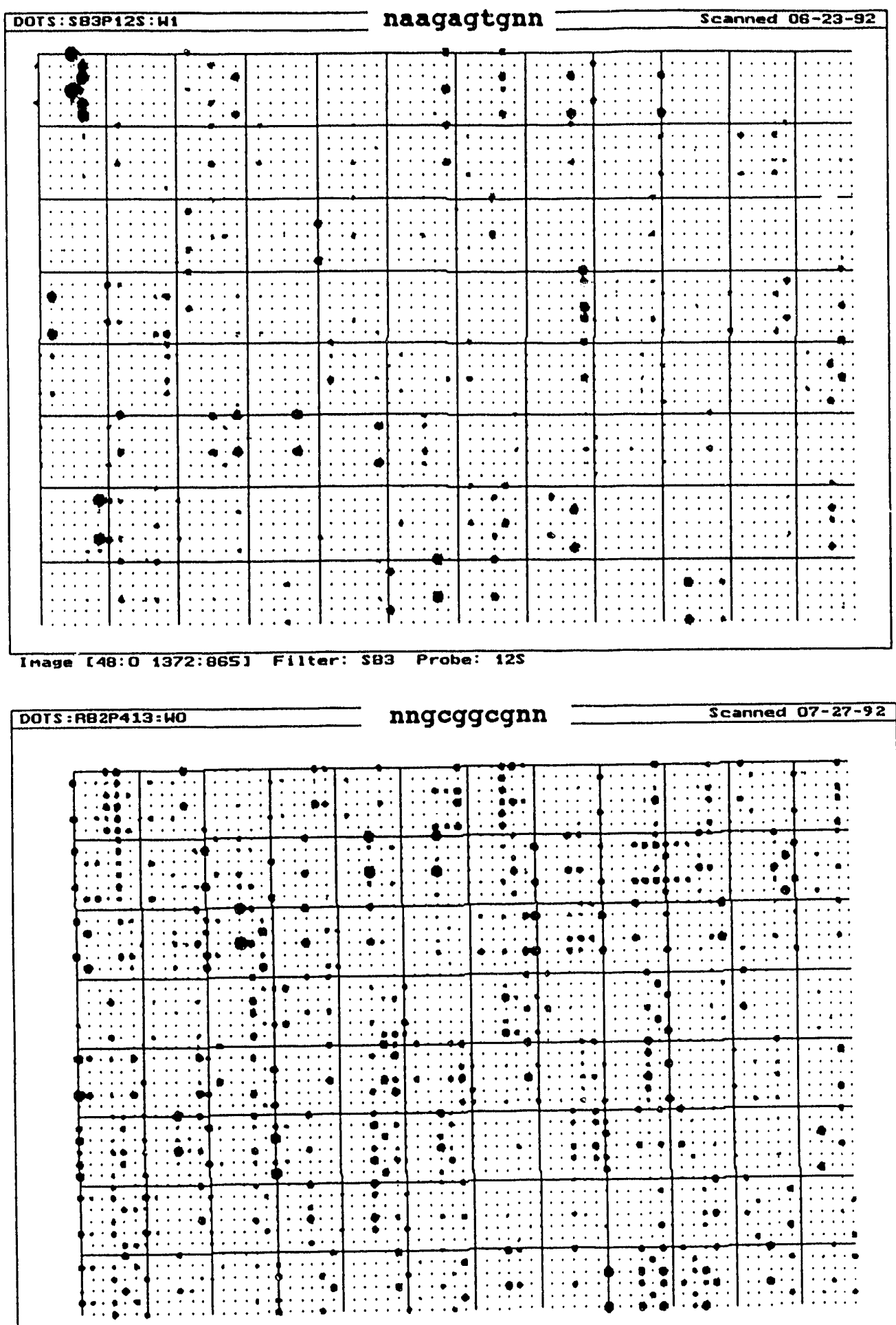

Image L0:0 1407:911] Filter: 82 Probe: 413 


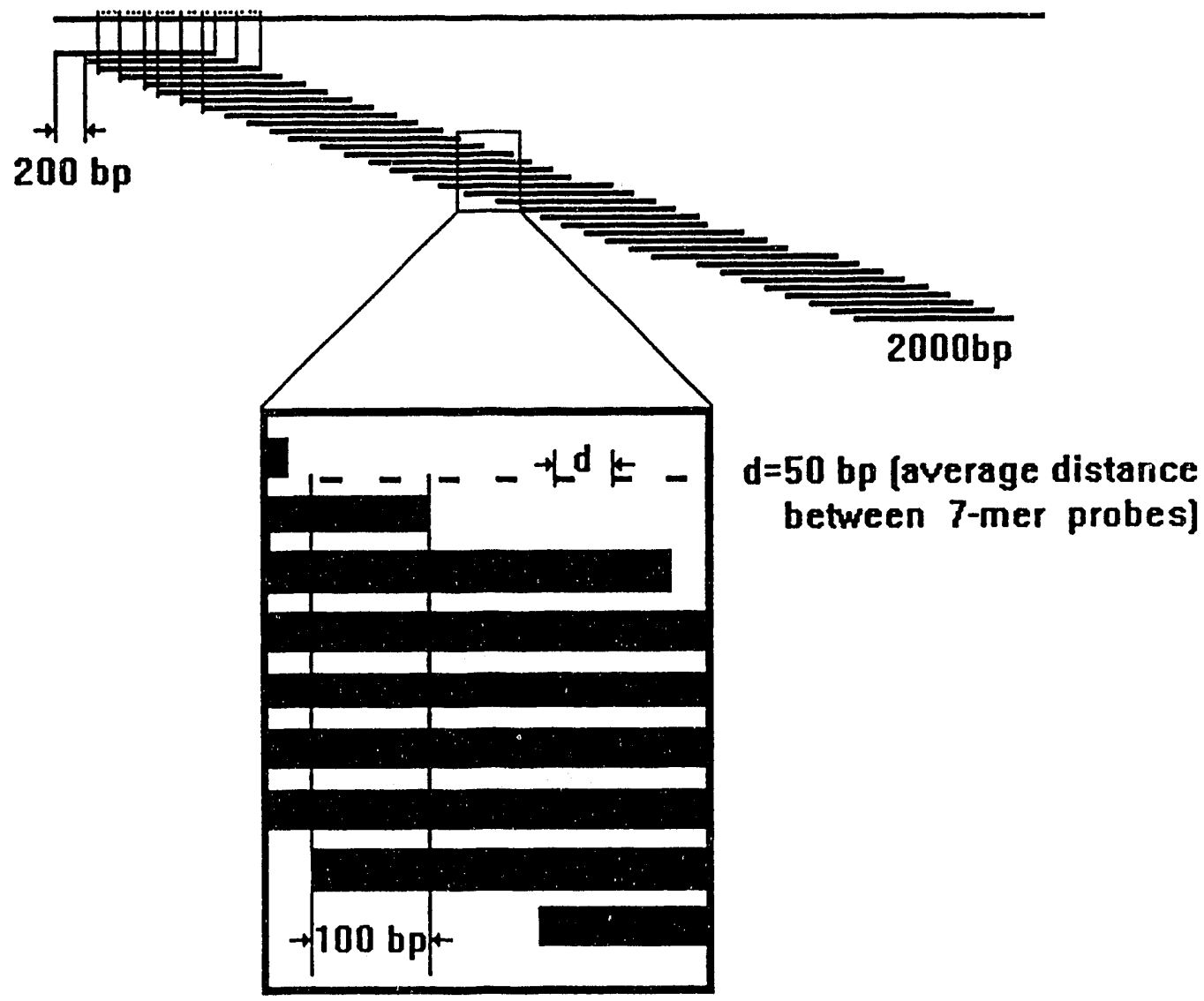




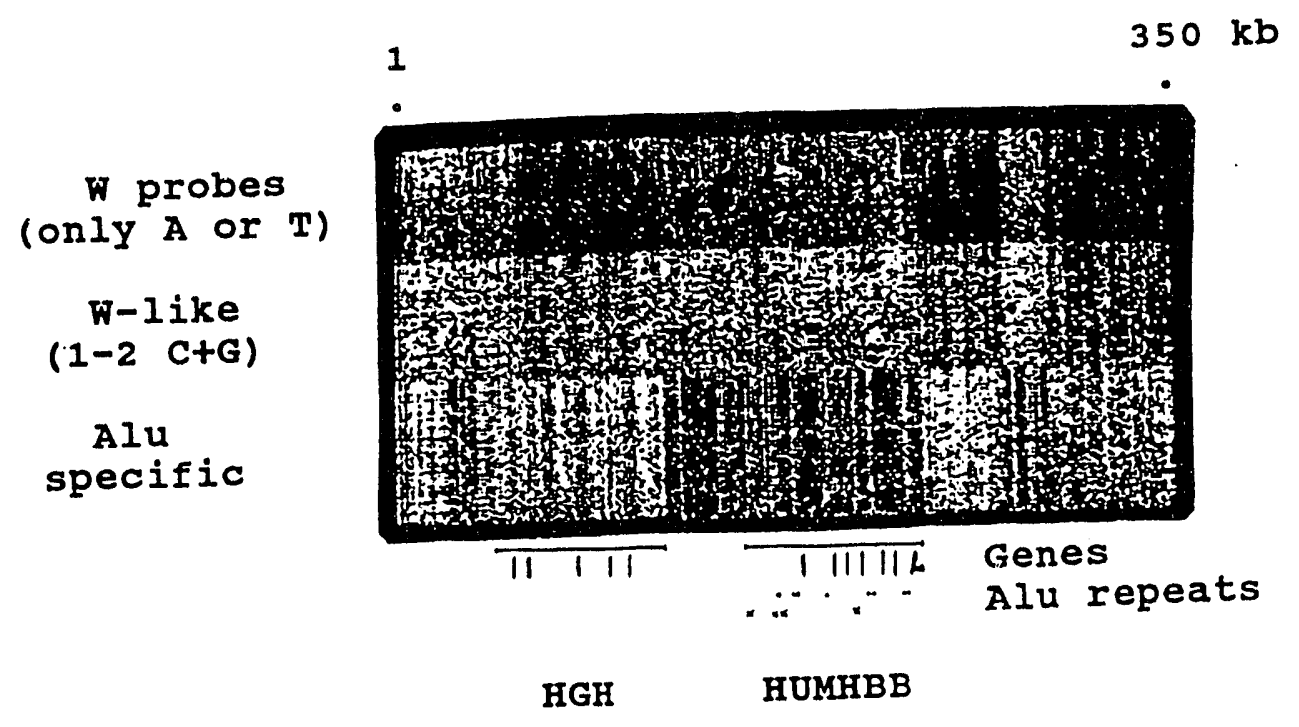

18 

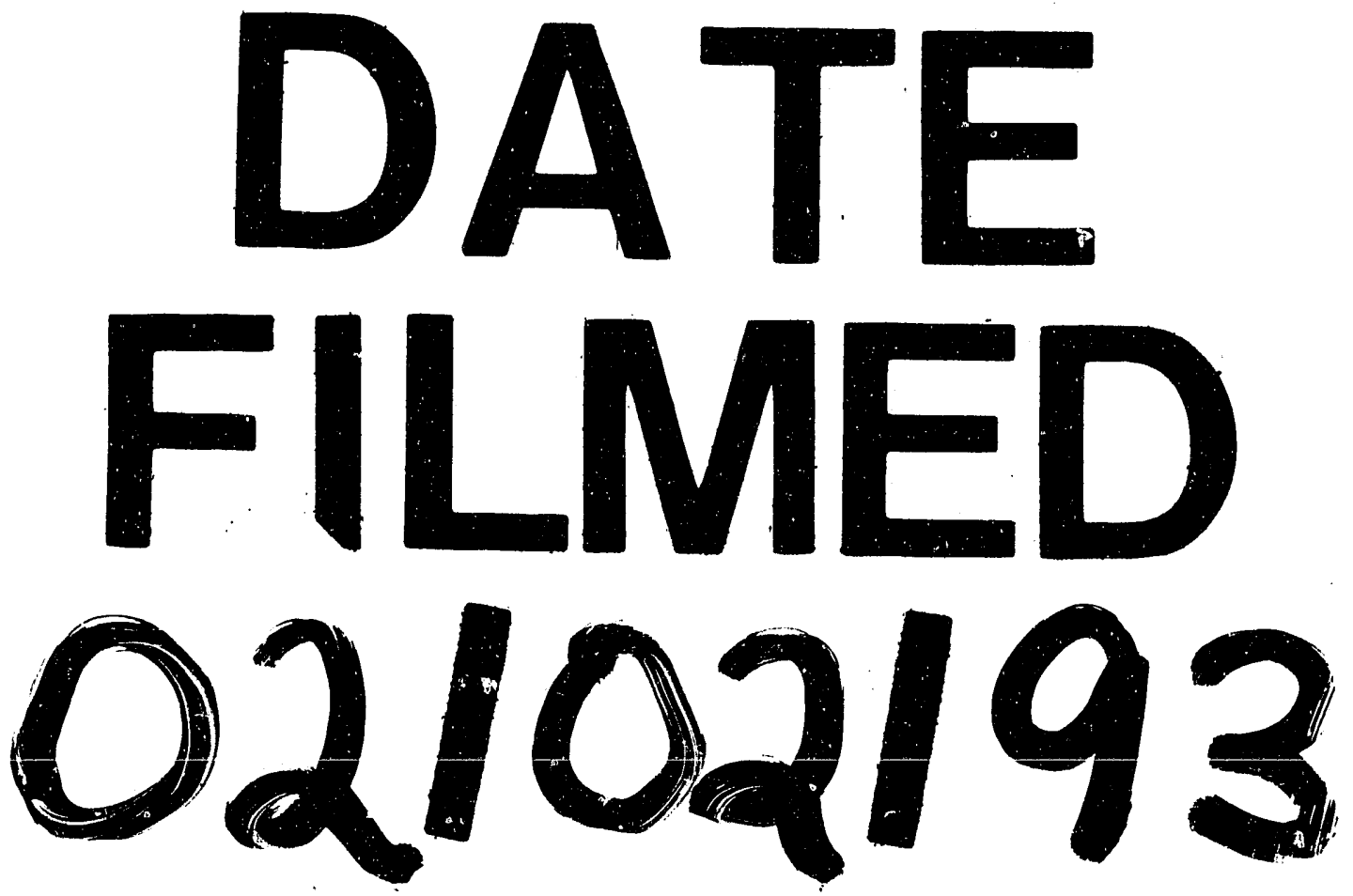
